\title{
MORTE E MORRER EM UNIDADE DE TERAPIA INTENSIVA PEDIÁTRICA: PERCEPÇÃO DOS PROFISSIONAIS DE SAÚDE
}

\author{
Maria Virgínia Martins Faria Faddul Alves ${ }^{1}$, Débora Nunes Scudeler², Claudia Helena Bronzatto Luppi³ \\ Maria José Trevizani Nitsche ${ }^{3}$, Lis Amanda Ramos Toso ${ }^{4}$
}

\begin{abstract}
RESUMO: O objetivo desta pesquisa descritiva foi conhecer a percepção dos profissionais de saúde que trabalham em Unidade de Terapia Intensiva Pediátrica em relação ao processo de morte e morrer. O estudo foi realizado no período de junho a agosto de 2011, os dados foram coletados por meio de entrevista estruturada e submetidos à análise estatística. Dos 25 participantes, $72 \%$ percebem a morte como um processo natural da vida, $60 \%$ sentem compaixão, sentimento que não interfere no cuidado ao paciente, $52 \%$ relataram não terem recebido preparo algum sobre o processo de morte e morrer e 76\% manifestaram interesse em realizar cursos de atualização sobre o tema. É necessária maior discussão acerca da formação acadêmica, e a oferta de atividades educativas e espaço para a troca de experiências, para que os trabalhadores possam melhor compreender e lidar com sentimentos e limitações perante a morte.
\end{abstract}

PALAVRAS-CHAVE: Morte; Terapia intensiva; Cuidados de enfermagem.

\section{DEATH AND DYING IN PEDIATRIC INTENSIVE CARE: THE HEALTH CARE PROFESSIONALS}

\begin{abstract}
The objective of this descriptive research was to investigate the perception of health care professionals who work in Pediatric Intensive Care Units in relation to the process of death and dying. The study was undertaken between June and August 2011, with data collected through structured interviews and subjected to statistical analysis. Of the 25 participants, $72 \%$ perceived death as a natural process of life, $60 \%$ felt compassion - a feeling that did not interfere in caring for the patient, $52 \%$ related that they had not received any preparation about the process of death or dying and $76 \%$ showed interest in taking a refresher course on the issue. Greater discussion is necessary about academic training, and the offer of educational activities and space for exchanging experiences, such that the workers may better understand and deal with feelings and limitations regarding death.
\end{abstract}

KEYWORDS: Death; Intensive care; Nursing care.

\section{MUERTE Y MURIR EN UNIDAD DE TERAPIA INTENSIVA PEDIÁTRICA: PERCEPCIÓN DE LOS PROFESIONALES DE SALUD}

RESUMEN: El objetivo de esta investigación descriptiva fue conocer la percepción de los profesionales de salud que trabajan en Unidad de Terapia Intensiva Pediátrica acerca del proceso de muerte y murir. El estudio fue realizado en el periodo de junio a agosto de 2011. Los datos fueron obtenidos por medio de entrevista estructurada y sometidos al análisis estadístico. De los 25 participantes, $72 \%$ perciben la muerte como un proceso natural de la vida, $60 \%$ sienten compasión, sentimiento que no interfiere en el cuidado al paciente, $52 \%$ relataron no haber recibido preparación alguna sobre el proceso de muerte y murir y $76 \%$ manifestaron interés en realizar cursos de actualización acerca del tema. Es necesario más discusión acerca de la formación académica, y la oferta de actividades educativas y espacio para el cambio de experiencias, a fin de que los trabajadores puedan comprender mejor y lidiar con sentimientos y limitaciones delante de la muerte.

PALABRAS CLAVES: Muerte; Terapia intensiva; Cuidados de enfermería.

${ }^{1}$ Enfermeira. Doutora em em Bases Gerais da Cirurgia. Professora do Departamento de Enfermagem da Faculdade de Medicina de Botucatu - UNESP.

${ }^{2}$ Graduanda do Curso de Enfermagem da Faculdade de Medicina de Botucatu - UNESP.

${ }^{3}$ Enfermeira. Doutora em Enfermagem. Professora do Departamento de Enfermagem da Faculdade de Medicina de Botucatu - UNESP. ${ }^{4}$ Gerente Técnica da UTI Pediátrica do Hospital das Clínicas da Faculdade de Medicina de Botucatu - UNESP.

Autor correspondente:

Recebido: $23 / 12 / 2011$

Maria Virgínia Martins Faria Faddul Alves

Aprovado: 01/08/2012

aculdade de Medicina de Botucatu - UNESP

R. Rubião Junior, SN - 18618-000 -Botucatu-SP-Brasil

E-mail: virginia@fmb.unesp.br 


\section{INTRODUÇÃO}

A morte significa a interrupção definitiva da vida e pode ser vivenciada tanto pelos seres humanos quanto pelos animais e plantas. Faz parte de um dos maiores mistérios da vida e ao longo da história demandou e ainda demanda muitos esforços para seu entendimento ${ }^{(1)}$. É uma experiência universal e sua representatividade pode variar entre as mais diversas culturas ${ }^{(2)}$, porém, o homem só a conhece através do processo de morrer de outras pessoas sem jamais ter a experiência direta com a morte e sua real dimensão(3).

Tempos atrás, acreditava-se que a morte ocorria quando o coração parava definitivamente. Atualmente, a morte biológica é caracterizada a partir da cessação das funções cerebrais ${ }^{(4)}$. Além do ponto de vista biológico, o ser humano caracteriza-se pelo aspecto simbólico, pelo ser que vive e convive com os demais e pelos valores que ele imprime às coisas e assim também é representada sua morte, pela ausência que fará no contexto familiar e social ${ }^{(5)}$.

A morte para alguns profissionais da saúde é sinônimo de não êxito. Quando ela ocorre, esses entendem como uma sensação ou sentimento de fracasso; consequência da formação acadêmica, pois os profissionais são instruídos a dar continuidade à vida e não são preparados para encarar a morte como um processo natural ${ }^{(5-6)}$. Antigamente, a pessoa morria em casa, ao lado da família, porém, com a institucionalização dos hospitais e Unidades de Terapia Intensiva (UTI), o processo de morte e morrer ocorre nos hospitais. Essas unidades proporcionam ao paciente o prolongamento da vida, através de tecnologia e medicamentos altamente eficazes ${ }^{(7-8)}$.

Os avanços científicos e as novas tecnologias, que promovem a manutenção da vida a todo custo, colocam os profissionais da saúde em situações estressantes e contraditórias, pois embora a recuperação do paciente seja o principal objetivo, ao se tornar iminente a morte, cabe a eles respeitar o momento, proporcionar todos os cuidados paliativos a seu alcance e tornarem o processo de morte e morrer o mais tranquilo e digno possível ${ }^{(9)}$. Em consequência, os profissionais de UTI vivenciam uma rotina repleta de dúvidas, instabilidades e procedimentos técnicos altamente complexos, pois, os pacientes internados nessas unidades, normalmente, são muito dependentes e seu estado de saúde é crítico ${ }^{(10-11)}$.

A morte de um paciente pode gerar, nos profissionais sentimentos de fracasso e frustração e quando é uma criança ou adolescente que morre, esses sentimentos podem ser potencializados, gerando angustia, sofrimento e dor pela morte precoce ${ }^{(12)}$. Ao cuidar de crianças e adolescentes, a chance do profissional de enfermagem criar proximidade e acabar apegando-se ao paciente é maior. Algumas vezes essa aproximação é intensa, gerando sentimento de empatia com a família ou então projeta a situação como se fosse seu próprio filho. Diante disso, os sentimentos de perda e a frustração podem ser potencializados provocando o luto, resultado do processo de morte ${ }^{(7,13)}$.

Quando o período de internação é muito longo, pode gerar um relacionamento mais intenso, pois é inevitável a aproximação do profissional com a criança, como se fosse um familiar. O vínculo afetivo é maior e está relacionado proporcionalmente com o tempo de internação da criança. No entanto, existem profissionais que, para evitar o sofrimento e a sensação de perda, limitam-se apenas aos procedimentos técnicos, evitando envolver-se emocionalmente com o paciente e isso pode prejudicar a assistência $^{(7,14)}$.

O objetivo deste estudo foi conhecer a percepção dos profissionais de saúde de uma UTI Pediátrica sobre o processo de morte e morrer. Este conhecimento poderá proporcionar melhores condições de trabalho aos profissionais, pois possibilitará reconhecer o processo de morte e morrer e, como trabalhar e vivenciar esse processo. Isto também irá corroborar com a qualidade da assistência prestada a esses pacientes.

\section{MÉTODO}

Trata-se de um estudo descritivo, desenvolvido na UTI Pediátrica de um hospital universitário. A unidade possui 7 leitos de internação, com média de permanência de quatro dias. Existem três horários de visita, com duração de duas horas e permitida a entrada de dois visitantes. A mãe ou acompanhante responsável pode entrar e permanecer junto ao paciente durante o horário que desejar.

A população foi composta por profissionais de saúde que atuam nessa unidade. O efetivo da unidade eram cinco enfermeiros, 14 técnicos e auxiliares de enfermagem, 12 médicos e residentes, num total de 31 profissionais. Todos os profissionais estavam aptos a participarem da pesquisa. Foram excluídos os profissionais que estavam em férias e/ou licença no período da coleta de dados, que foi realizada entre junho a agosto de 2011, por meio de entrevista estruturada.

Os profissionais da unidade eram abordados pela entrevistadora, que lhes explicava a finalidade da pesquisa e, quando concordavam em participar, era marcado horário para a realização da entrevista e eles assinavam o Termo de Consentimento Livre e Esclarecido e a entrevista era realizada em uma sala da UTI Pediátrica. A análise estatística 
foi realizada através da frequência absoluta e/ou relativa e em porcentagem dos dados quantitativos. A pesquisa foi aprovada pelo Comitê de Ética em Pesquisa da Faculdade de Medicina de Botucatu, Of. 84/11-CEP.

\section{RESULTADOS}

Dos 31 profissionais que atuam na UTI Pediátrica, 25 $(80,6 \%)$ responderam à entrevista, sendo $24 \%$ enfermeiras (06), 16\% auxiliares de enfermagem (04), 24\% técnicas em enfermagem (06) e 36\% médicos e residentes (09). Os demais profissionais que não responderam ao questionário (19,4\%) estavam em licença médica, sem tempo disponível ou recusaram-se a participar.

A idade variou entre 25 e 51 anos e a população consultada (25) foi predominantemente feminina (88\%). O tempo de formação verificado entre os $48 \%$ dos profissionais foi de 1 a 5 anos. Os dados estão demonstrados no gráfico 1 .

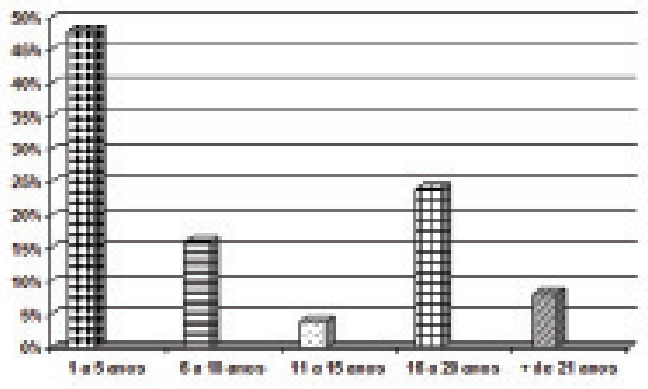

Gráfico 1 - Distribuição do tempo de formado dos profissionais da Unidade de Terapia Intensiva Pediátrica. Botucatu, 2011

Em relação ao tempo de trabalho na UTI Pediátrica, foi verificado que $32 \%$ dos profissionais tinham menos de um ano de formados e $24 \%$ tinham de um a cinco anos de formados. Todos os dados estão demonstrados no gráfico 2 .

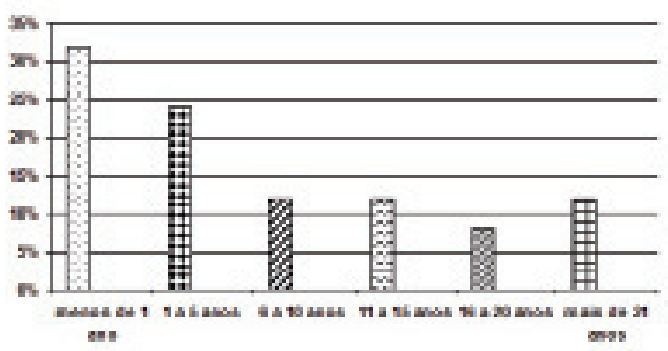

Gráfico 2 - Distribuição do tempo de trabalho dos profissionais na Unidade de Terapia Intensiva Pediátrica. Botucatu, 2011
Os profissionais de saúde foram questionados sobre sua opinião em relação à morte. A morte é um processo natural da vida para $72 \%$ dos entrevistados. Os dados estão demonstrados no gráfico 3.

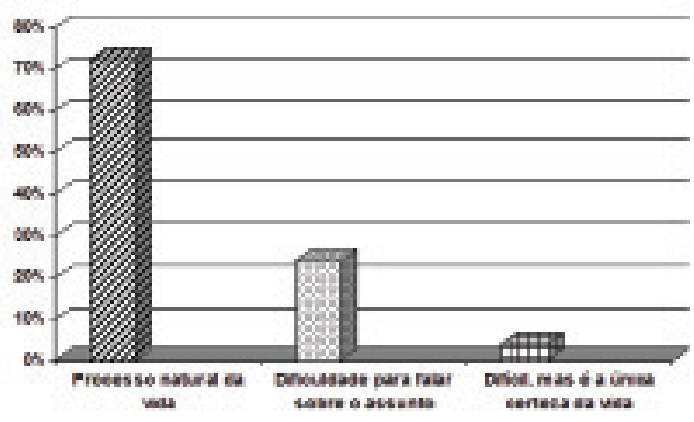

Gráfico 3 - Distribuição da opinião dos profissionais da Unidade de Terapia Intensiva Pediátrica em relação à morte. Botucatu, 2011

Ao cuidar de um paciente em fase terminal, podem surgir diversos sentimentos e por isso perguntamos aos profissionais como se sentem ao cuidar de um paciente em processo de morte e morrer. As respostas foram: sentem compaixão, mas, esse sentimento não interfere na forma como cuidam do paciente para 15 profissionais (60\%); 20\% (05) gostam de cuidar desse tipo de paciente, pois se sentem bem; $12 \%$ (03) afirmam ser indiferente ao fato do paciente estar em fase terminal, $o$ cuidado é o mesmo de qualquer outro paciente; $4 \%$ (01) apresentam compaixão, dó e isso interfere no cuidado e $4 \%$ (01) cuidam com todo respeito que um paciente pode ter nessa fase tão difícil.

Foi questionado se durante o curso de formação, o profissional teve informações, aulas ou cursos sobre o cuidar do paciente no processo de morte e morrer. Foi verificado que $48 \%$ dos entrevistados afirmaram ter vivenciado, de alguma maneira, o processo de morte e morrer, seja através de discussões em grupo com psicóloga e psiquiatra; filmes e trabalhos; apoio psicológico individual; terapia antálgica; cursos extracurriculares ou aulas sobre cuidados paliativos e comunicação com familiares. No entanto, a maior parte $(52 \%)$ relatou não ter recebido preparo algum durante o período de formação sobre o cuidador do paciente no processo de morte e morrer, vivenciado no início das atividades profissionais.

Quando foram questionados sobre seu interesse em participar de cursos de atualizações sobre o tema, 76\% (19) responderam que sim e a justificativa foi dizer que a assistência ao paciente inclui todo o ciclo da vida, desde o nascimento até a morte, e é necessário que o 
profissional da saúde, principalmente os profissionais que atuam em UTI, estejam preparados para lidar com a finitude do ser humano. Disseram também que quanto mais instruídos, mais aptos estarão para cuidar do paciente e saber como agir perante a família que acompanha e que necessita de informações. Os $24 \%$ restantes disseram não se interessarem por cursos de atualização, devido à falta de tempo, e também, por entenderem que o assunto morte é polêmico e doloroso.

\section{DISCUSSÃO}

O perfil dos profissionais é, em sua maioria, do sexo feminino, com formação entre um a cinco anos e, consequentemente, com tempo inferior a cinco anos de trabalho na unidade. Assim, esses dados apontam para um grupo composto por profissionais com formação recente, porém que atuam em um ambiente onde estão mais suscetíveis a cuidar de pacientes em processo de morte e morrer, dando-lhes vasta experiência e oportunidades de enfrentamento.

No presente estudo foi verificado que a maioria dos entrevistados caracteriza a morte como um processo natural e, assim como nascer, morrer faz parte da existência humana. Outra pesquisa relata que, apesar da conotação negativa que a morte recebe e os sentimentos de fracasso e impotência que podem ser vividos perante ela, do ponto de vista biológico, a morte é algo extremamente natural ${ }^{(5)}$. Ao longo da vida sofremos várias perdas e mudanças que caracterizam o fim de algo que vivemos, porém a morte torna-se a última perda no decorrer do desenvolvimento humano ${ }^{(4)}$, e por isso, para alguns profissionais de saúde entrevistados neste estudo, era difícil falar sobre o assunto.

A justificativa desses profissionais sobre a dificuldade de lidar com esse assunto é intensificada com o contato prolongado com o paciente, devido a suas idas e vindas ao hospital, possibilitam o laço afetivo entre os profissionais, familiares e pacientes. O sofrimento é inevitável para algumas pessoas, no entanto, este tabu criado em torno da morte as impede de expressar seus sentimentos reais ${ }^{(15)}$, e assim, os profissionais apresentam dificuldades de falar sobre o assunto, como foi observado neste trabalho.

Na literatura e também neste estudo foi constatado que os profissionais sentem compaixão pelos pacientes, porém cuidam deles da melhor forma possível, sem deixar que esse sentimento interfira no cuidado prestado. Existem aqueles que gostam de prestar atendimento a pacientes em fase terminal, ou fora das possibilidades terapêuticas, por entenderem que é nessa fase que precisam de maior atenção, compreensão e respeito. Ressalta-se que o sentimento de compaixão surge entre a equipe, principalmente com pacientes que passam por longos períodos de sofrimento e não possuem bons prognósticos. A compaixão pode se unir ao desejo de morte desse paciente pelo profissional, a fim de que o paciente encontre o descanso e fique livre do sofrimento, mas ao mesmo tempo, o profissional apresenta o sentimento de culpa e revolta por ter esses sentimentos $^{(3)}$. O vínculo emocional e a empatia com esses pacientes são inevitáveis e imprescindíveis para o cuidado adequado, garantindo que todas as necessidades sejam supridas. Porém, deve-se ter cuidado e respeitar os próprios limites ${ }^{(14)}$.

Essa mistura de sentimentos pode ou não interferir na assistência a esse paciente, pois, com os avanços tecnológicos, houve também o aumento da expectativa em relação à morte, e cada vez mais, é possível interferir e adiar o fim da vida de acordo com as determinações e possibilidades encontradas pela equipe ${ }^{(16)}$.

Outro fator que pode, ou não, interferir na assistência a esse paciente é que, nas UTI Pediátricas, a responsabilidade em relação à vida é maior, pois uma criança está apenas no início da vida e sua morte gera maior sofrimento entre a equipe. Com o tempo, barreiras para o enfrentamento da morte são criadas pelos profissionais. Embora cada um crie de maneira distinta suas barreiras, de uma forma geral os funcionários com maior tempo de atuação profissional enfrentam a situação de forma mais preparada ${ }^{(15)}$. Este fato não foi verificado neste estudo, pois foi observado que muitos profissionais que atuam na UTI Pediátrica têm menos de cinco anos de formados e também de experiência na unidade.

Por meio das respostas obtidas, foi observado que a maioria dos profissionais não recebeu preparo algum para este tipo de situação durante a sua formação, e assim, adquiriu a experiência de lidar com a morte e o morrer durante a sua vida profissional. Também aprendeu a melhor maneira de cuidar, tanto de si próprio, como para o cuidado com os pacientes.

De acordo com a literatura, a melhor maneira de suprir as necessidades geradas em torno do processo de morte e morrer é realizar abordagens com maior freqüência sobre o tema ${ }^{(15)}$. Isto também é relatado em estudo feito com estudantes de enfermagem, onde a conclusão foi de que há necessidade de se criar espaços para discussões sobre o assunto (morte e morrer) no decorrer das atividades curriculares, possibilitando 
momentos de reflexão do estudante para melhor compreensão e enfrentamento dessas situações ${ }^{(16)}$. Este assunto precisa ser encarado de maneira positiva e pró-ativa por parte de docentes, enfermeiros e alunos de graduação, para que seja possível a formação de profissionais com capacidade de enfrentar e discutir a vivência da morte dos pacientes ${ }^{(9)}$.

É importante que as escolas de enfermagem incluam o tema morte e morrer ao longo do curso, para que haja melhora da assistência prestada pelos alunos e que possibilitem a formação de profissionais mais seguros sobre a temática ${ }^{(17)}$. Os profissionais de enfermagem sentem-se frágeis para atuar junto ao paciente fora das possibilidades terapêuticas e que existe uma lacuna entre a formação do profissional e a manutenção de treinamento pelas instituições de saúde.

Diante de um prognóstico ruim, ou de um quadro irreversível de uma criança/adolescente, os profissionais de saúde necessitam de preparo para compreender e agir da melhor maneira possível diante do sofrimento vivenciado durante este processo de terminalidade. Ao desvincular a morte do constrangimento e sentimento de culpa e fracasso, através de planejamentos, discussões e estudos ao longo da formação profissional, podemos ampliar o cuidado dando ao paciente que está sem possibilidade de cura a atenção necessária a ele nesse processo de enfrentamento ${ }^{(18)}$.

Estudos sobre a morte e o morrer com os profissionais da saúde mostram a importância da abordagem do tema morte entre os profissionais de saúde. O conhecimento do assunto fortifica a assistência ao paciente terminal. Porém, ainda existe a questão em saber se as pessoas realmente desejam ser preparadas para este tipo de situação, e como desejam que isso aconteça. Como constatamos através deste estudo, alguns profissionais sentem necessidade de uma abordagem mais freqüente da morte e de saber lidar com a carga de estresse que a situação pode gerar.

É preciso também aprimorar a forma de cuidado com os pacientes, tratando-os da maneira mais digna possível e para sentirem-se seguros quanto à família, que também precisa de apoio e compreensão. Um estudo ressalta que o tema pode gerar nas pessoas inúmeros questionamentos de naturezas distintas e torna-se ainda mais significante $\mathrm{e}$ urgente aos profissionais da área da saúde, pois convivem diariamente com o risco iminente de morte. Inúmeras são as tentativas de respostas por parte da ciência, religião, crença, filosofia e outros, porém nenhuma é soberana e abrange a universalidade. Torna-se necessário encará-la como questão multidisciplinar, abordar o tema através do desenvolvimento de pesquisas, cursos de atualizações, criação de espaço para debates e troca de experiências, aprofundar o tema, tanto nas universidades e cursos de formação profissional, como nos hospitais e demais instituições de saúde ${ }^{(19)}$.

\section{CONSIDERAÇÕES FINAIS}

Neste estudo o objetivo principal foi conhecer a opinião dos profissionais de saúde que trabalham em uma UTI Pediátrica em relação ao processo de morte e morrer. Foi possível identificar que, apesar do tabu que envolve o processo de morte e morrer, os profissionais que atuam nessa unidade, procuram encará-la como forma natural da vida e mesmo com diversos sentimentos envolvidos nesse processo, cada caso deve ser vivenciado de maneira individual. A intensidade do sofrimento depende do tempo de internação e grau do vínculo estabelecido como a criança no processo de cuidar. Porém, busca-se tratar do paciente em processo de morte da mesma maneira que de qualquer outra criança, sem diferenciá-la devido à sua terminalidade.

Faz-se necessário melhorar e estimular reflexões, discussões e conhecimentos sobre o assunto, desde o aluno de enfermagem até o profissional, para que essa temática seja vivenciada, abordada e trabalhada pelos profissionais de saúde.

Através desta pesquisa, foi possível visualizar uma proposta para essa unidade, de cursos de educação continuada sobre o assunto morte e morrer para os profissionais de saúde, a fim de melhorar as condições de trabalho e assistência aos pacientes da UTI Pediátrica.

\section{REFERÊNCIAS}

1. Dastur F. A morte, ensaio sobre a finitude. Rio de Janeiro: Difel, 2002.

2. Martins G. Laços atados - a morte do jovem no discurso materno. Curitiba: Moinho do verbo, 2001.

3. Palú LA, Labronici LM, Albini L. A morte no cotidiano dos profissionais de enfermagem de uma unidade de terapia intensiva. Cogitare enferm. 2004;9(1):33-41.

4. Brêtas JRS, Oliveira JR, Yamaguti L. Reflexões de estudantes de enfermagem sobre morte e o morrer. Rev. Esc. Enferm. USP. 2006;40(4):477-83.

5. Combinato DS, Queiroz MS. Morte: uma visão psicossocial. Estudos de Psicologia. 2006;11(2):209-16. 
6. Souza LB, Souza LEEM, Souza AMA. A ética durante o processo de morrer: relato de experiência. Rev. bras. enferm. 2005;58(6):731-4.

7. Costa JC, Lima RAG. Luto da equipe: revelações dos profissionais de enfermagem sobre o cuidado à criança/ adolescente no processo de morte e morrer. Rev. LatinoAm. enfermagem. 2005;13(2):151-7.

8. Boemer MR. O fenômeno da morte: o pensar, o conviver e o educar. [tese]. Riberão Preto (SP): Universidade de São Paulo; 1989.

9. Bellato R, Araújo AP, Ferreira HF, Rodrigues PF. A abordagem do processo do morrer e da morte feita por docentes em um curso de graduação em enfermagem. Acta paul. enferm. 2007;20(3):255-63.

10. Paschoa S, Zanei SSV, Whitaker IY. Qualidade de vida dos trabalhadores de enfermagem de unidades de terapia intensiva. Acta paul. enferm. 2007;20(3):305-10.

11. Mark BA, Hagenmueller AC. Technological and environmental characteristics of intensive care units. Implications for job redesign. J. Nurs. Adm. 1994;24(4 Suppl):65-71.

12. Spíndola T, Macedo MCS. A morte no hospital e seu significado para os profissionais. Rev. bras. enferm. 1994;47(2):108-17.

13. Bromberg MHPF. A psicoterapia em situação de perdas e luto. Campinas: Livro Pleno; 2000.

14. Lunardi WDFilho, Sulzbach RC, Nunes AC, Lunardi VL. Percepções e condutas dos profissionais de enfermagem frente ao processo de morte e morrer. Texto Contexo Enferm. 2001;10(3):60-81.

15. Shimizu HE. Como os trabalhadores de enfermagem enfrentam o processo de morrer. Rev. bras. enferm. 2001;60(3):257-62.

16. Vargas D. Morte e morrer: sentimentos e condutas de estudantes de enfermagem. Acta paul. enferm. 2010;23(3):404-10

17. Lana SO, Passos ABB. Preparo dos acadêmicos de enfermagem no processo de morte e morrer. Revista Enfermagem Integrada - Ipatinga. 2008;1(1):80-90.

18. Oliveira SG, Quintana AM, Bertolino KCO. Reflexões acerca da morte: um desafio para a enfermagem. Rev. bras. enferm. 2010;63(6):1077-80.
19. Kovácx MJ. Educação para a morte. Psicol. cienc. prof. São Paulo 2005;25(3):484-97. 